\title{
Comparison of SARS-CoV-2 antigen electrochemiluminescence immunoassay to RT-PCR assay for laboratory diagnosis of COVID-19 in Peshawar
}

https://doi.org/10.1515/dx-2021-0078

Received June 4, 2021; accepted August 4, 2021;

published online August 30, 2021

\section{Abstract}

Objectives: Antigen based rapid diagnostic tests possesses a potential to be utilized along with Gold standard methods to detect Covid-19 infection to cope with the demand of testing. The aim of this study was to determine diagnostic accuracy of electrochemiluminescence based automated antigen detection immunoassay comparing with molecular based test RT-PCR (Covid-19).

Methods: It was a cross-sectional study conducted in RMI Peshawar, from 1st April 2021 till 30th April 2021. The study comprised 170 individuals who were suspected of having Covid-19. Nasopharyngeal samples taken from suspected individuals were analyzed by RT-PCR and automated antigen test (Elecsys SARS-CoV-2 Antigen) simultaneously. The correlation of SARS-CoV-2 antigen with PCR positive and negative cases was analyzed for specificity, sensitivity respectively.

Results: The ECLIA based Elecsys antigen test (Roche) revealed overall sensitivity $72 \%$, specificity $95 \%$ and accuracy of $94.9 \%$. Sensitivity of antigen test progressively declined from $94.3 \%$ in $\mathrm{Ct}<25$ to $70.8 \%$ in $\mathrm{Ct} 26-29$ and then to $47.2 \%$ in $\mathrm{Ct} 30-35$.

Conclusions: Based on the findings of our study we conclude that automated antigen testing (Elecsys SARS-CoV-2 Antigen) cannot replace molecular based testing like RT PCR. Elecsys SARS-CoV-2 Ag test should be used complementary to RT-PCR in testing algorithms. Frequent testing strategy should be

*Corresponding author: Bilal Iqbal, MBBS, Resident Chemical Pathology, Rehman Medical Institute Peshawar, Peshawar, Pakistan, E-mail: bilaliqbal4124@gmail.com

Maria Khan, Noman Shah, Mirza Muhammad Dawood and Valeed Jehanzeb, Rehman Medical Institute Peshawar, Peshawar, Pakistan Mohsin Shafi, Khyber Medical College Peshawar, Peshawar, Pakistan adopted while using automated antigen testing to overcome its limitation in individuals with low viral loads.

Keywords: COVID-19; electrochemiluminescence immunoassay; rapid diagnostic testing; RT-PCR; SARS-CoV-2 antigen.

\section{Introduction}

SARS coronavirus 2 (SARS-CoV-2) emerged in 2019 as a novel coronavirus from the same family as SARS-CoV and Middle East Respiratory Syndrome (MERS) coronavirus (CoVID-19). Later, World Health Organization (WHO) declared it a pandemic on March 11, 2020 [1]. As of present, the total number of SARS-CoV-2 positive cases in the world is 171,222,477 and have been climbing vigorously. The SARS-CoV-2 is an RNA virus having four key structural proteins that include nucleocapsid, spike protein, core matrix protein and envelop. It uses the spike protein for binding with the receptor, angiotensin-converting enzyme 2, which expresses itself on epithelial stem cells type 2 (Alveolar) of lungs, and other tissues of arteries, veins, kidneys and salivary glands [2]. Early diagnosis plays vital role in treatment of the disease and its containment, for which continuous efforts were made to establish rapid, accurate and affordable diagnostic test for diagnosis of SARS-CoV-2 infection [3]. Predominantly SARS-CoV-2 diagnostic tests were mostly based on its molecular detection, which has a high sensitivity and specificity [4]. Undoubtedly RT-PCR for CoVID-19 is a standard and most commonly used diagnostic tool for detection of viral RNA [5] it requires specialized laboratory instruments and trained personals. Overall, with current low capacity testing of RT-PCR, there is apparent delayed diagnosis and clinical management, eventually leading to propagation of the disease [6]. However, detecting a large number of patients through RT-PCR in shorter duration during a pandemic is a difficult task that may potentially involve technical or budgetary challenges. For resource-constrained countries 
like Pakistan, rapid, reliable, and affordable diagnosis of SARS-CoV-2 is required to meet national or international needs [7]. Multiple rapid antigen tests gained emergency authorization for diagnosis of acute respiratory syndrome. In spite of the fact that rapid antigen testing is user-friendly, economical and safe at point-of-care clinical performance and validation could be a source of concern [8]. In order to develop new testing approach to combat the unprecedented CoVID-19 epidemic we can evaluate the diagnostic accuracy of the suitable diagnostic tests to the gold standard molecular based assay. In Pakistan, there is a pressing need to design a viable algorithm that properly fulfill the nationwide requirement for extended SARS CoV-2 screening, diagnosing, and treating in a timely manner. This study was conducted to compare automated antigen (Roche Elecsys SARS-CoV-2 Ag) electrochemiluminescence immunoassay based SARS-CoV-2 diagnostic assay to molecular based tests RT-PCR in order to validate a rapid SARS-CoV-2 testing strategy for suspected patients visiting Rehman Medical Institute, Peshawar.

\section{Materials and methods}

A cross-sectional study was conducted from 1st April to 30th April at Pathology department of Rehman Medical Institute (RMI), Peshawar, Pakistan. In Peshawar total number of recorded Covid-19 cases till 30th April 2021 was 45,808 with 1,680 deaths [9]. Subjects were selected by using nonprobability convenient sampling technique. Patients of both genders were included in study. Informed consent was taken from all the patients. The study was approved by the Ethics Committee of Rehman Medical Institute, Peshawar. Nasopharyngeal swab specimens in a single viral-transport medium tube were obtained under transmission-based precautions from patients presenting at screening clinic. To examine the diagnostic accuracy, we used samples from 170 patients that were tested on RT-PCR and automated antigen detection assay (Roche Elecsys SARS-CoV-2 Ag) simultaneously. Participants in the study were patients referred from both IPD (Inpatient department) and OPD (Outpatient Department) of RMI. Selection criteria for enrollment, those patients were enrolled who were symptomatic and came to RMI for testing and treatment. A Real-time RT-PCR assay was used for the quantitative detection of ribonucleic acid (RNA) Covid-19 (TibMol Biol Roche Ltd Real-Time PCR assay), in nasal and throat swabs from suspected patients. The product kit had oligonucleotide primers and hydrolysis probes (dual-labeled) (TaqMan Technology), control medium, along with manual nucleic acid extraction (HighPure Complete DNA and RNA Purification Kit, Roche $\mathrm{GmbH})$. The oligonucleotide probe and primers were selected from the orf1 ab genome region and also targeting RNA dependent RNA polymerase - RdRp of SARS-CoV-2. RNA extracted and from nasal and oropharyngeal swabs was reverse transcribed to cDNA and successively amplified by real-time PCR instruments: Bio-Rad CFX-96 CT1000 (3.1 version). Temperature for amplification was $50^{\circ} \mathrm{C}$ for $17 \mathrm{~min}, 95^{\circ} \mathrm{C}$ for 5 min, subsequently 45 cycles of $95^{\circ} \mathrm{C}$ for $17 \mathrm{~s}$ and $60{ }^{\circ} \mathrm{C}$ for $35 \mathrm{~s}$. The concentration of 100 copies $/ \mu \mathrm{L}$ whole viral genome RNA of SARS-CoV-2 was the limit of detection of this assay. Specimens were processed and results were dispatched within $6-8 \mathrm{~h}$, if delay was expected then specimens were to be stored up to $72 \mathrm{~h}$ at $2-8^{\circ} \mathrm{C}$ after collection. The extracted RNA was preserved at $-70{ }^{\circ} \mathrm{C}$ freezer for long term storage. To ensure the integrity of RT-PCR results, an internal control was tested for each patient simultaneously, as well as negative and positive controls. Samples having exponential growth curve and CT value of $\leq 35$ were considered confirmed positive.

Next, automated antigen by Roche Switzerland (Elecsys SARS-CoV-2) electrochemiluminescence immunoassay "ECLIA" was performed that uses antibodies coated with microparticles against the target nucleocapsid antigen of SARS-CoV-2, with TAT of almost 15-18 min. In the first step $30 \mu \mathrm{L}$ of the sample is mixed with biotinylated monoclonal antiSARS-CoV-2 antibodies, and monoclonal anti-SARS-CoV-2-Ag antibodies labeled with Ruthenium. In second step streptavidin-coated microparticles is added to the solution, the resulting mixture binds to the solid. The reaction mixture is transferred to measurement cell, where the microparticles are magnetized to the electrode surface. This reaction emits signals (chemiluminescent emission) which is proportional to amount of antigen present in sample. Results are generated automatically by software in the form of cut-off index reactive $(>1.0)$ and Non-reactive $(<1.0)$. Limit of detection of SARS-COV-2 Ag assay $22.5 \mathrm{TCID} 50 / \mathrm{mL}$ [10]. Statistical analysis was done with SPSS version 20. The relationship between automated antigen testing and RT-PCR was investigated in order to derive automated antigen assay diagnostic accuracy (sensitivity, and specificity). RT-PCR was used as the gold standard to calculate sensitivity and specificity with $95 \%$ confidence intervals ( $95 \% \mathrm{CI})$.

\section{Results}

This study included 170 suspected CoVID-19 patients. Demographic (age, sex) and clinical record of the study population were obtained from the record section RMI in PCR (Covid-19) positive patients. Most common symptoms were cough (60\%), followed by fever (40\%), shortness of breath $(20 \%)$, loss of smell (20\%) and body aches (30\%).

Additionally, in order to segregate positive cases (RT-PCR) on the basis of viral load we obtained the Cycle threshold (Ct) value. In RT-PCR, this value represents the point at which amplification begins and is inversely related to the viral load in these samples (high viral loads is indicated by lower Ct values) [11]:

Among total cases, 48\% (83) were males, 52\% (87) females, having male to female ratio of 0.92 . The mean age was 45 years (range 14-89 years).

Out of 127 RT-PCR positive samples 92 samples tested positive and 35 samples tested negative by automated Elecsys SARS-CoV-2 Antigen. Out of total 43 RT-PCR negative samples 42 tested negative and 1 tested positive by Elecsys SARS-CoV-2 Antigen. In overall samples sensitivity of Elecsys SARS-CoV-2 Antigen was 72.4\% (95\% confidence interval: $62.4-78.8 \%$ ) and specificity was $95 \%$ (CI: 83.0-99.4\%) (Table 1).

Next, we examined the relationship of viral loads that is expressed in terms of cycle threshold $(\mathrm{Ct})$ value in $\mathrm{RT}$ PCR 
Table 1: Demographic information of symptomatic patients (COVID-19).

\begin{tabular}{|c|c|}
\hline Variables & Frequency $n=170, n(\%)$ \\
\hline \multicolumn{2}{|l|}{ Gender } \\
\hline Male & $83(48)$ \\
\hline Female & $87(52)$ \\
\hline \multicolumn{2}{|l|}{ Age, years } \\
\hline $14-30$ & $46(27)$ \\
\hline $31-45$ & $50(29)$ \\
\hline $45-60$ & $37(21)$ \\
\hline$>60$ & $37(21)$ \\
\hline \multicolumn{2}{|l|}{ Sign and symptoms } \\
\hline Cough & $102(60)$ \\
\hline Short of breath & $34(20)$ \\
\hline Fever & $68(40)$ \\
\hline Body aches & $51(30)$ \\
\hline Loss of smell & $34(20)$ \\
\hline \multicolumn{2}{|l|}{ Onset of symptoms, days } \\
\hline Presented during 1-3 & $58(34)$ \\
\hline $4-6$ & $48(28)$ \\
\hline$>6$ & $64(38)$ \\
\hline
\end{tabular}

with antigen assay. We divided the RT PCR positive samples based on Ct value into three groups: Ct $<25,25-29$, 30-35 and derived sensitivity in each group respectively. Table 2 summarizes the distribution of $\mathrm{Ct}$ values and sensitivity of Elecsys SARS-CoV-2 Antigen in each group separately. Sensitivity of Elecsys SARS-CoV-2 Antigen gradually decreases when viral load decreases (Table 3).

Table 2: Detection of SARS-CoV-2 by Elecsys SARS-CoV-2 antigen and Covid-19 RT-PCR.

\begin{tabular}{llrr}
\hline & \multicolumn{2}{c}{ Covid-19 RT-PCR } \\
\cline { 3 - 4 } & & Detected & Not detected \\
\hline Elecsys SARS-CoV-2 antigen & Positive & 92 & 1 \\
& Negative & 35 & 42 \\
\hline
\end{tabular}

Table 3: Diagnostic performance of the evaluated automated antigen test according to viral load.

\begin{tabular}{|c|c|c|c|c|}
\hline $\begin{array}{l}\mathrm{Ct} \\
\text { value }\end{array}$ & $\mathbf{n}$ & Accuracy & $\begin{array}{r}\text { Sensitivity }(95 \% \\
\mathrm{Cl})\end{array}$ & $\begin{array}{l}\text { Specificity (95\% } \\
\mathrm{Cl})\end{array}$ \\
\hline Overall & 170 & $\begin{array}{l}94.9 \% \\
(90.4-97.7 \%)\end{array}$ & $\begin{array}{r}72.4 \% \\
(62.4-78.8 \%)\end{array}$ & $\begin{array}{l}97.7 \% \\
(87.7-99.9 \%)\end{array}$ \\
\hline$<25$ & 53 & $\mathrm{~N} / \mathrm{C}$ & $\begin{array}{r}94.3 \% \\
(84.3-98.8 \%)\end{array}$ & $\mathrm{N} / \mathrm{C}$ \\
\hline $25-29$ & 38 & $\mathrm{~N} / \mathrm{C}$ & $\begin{array}{r}70.8 \% \\
(55.9-83.0 \%)\end{array}$ & $\mathrm{N} / \mathrm{C}$ \\
\hline $30-35$ & 36 & $\mathrm{~N} / \mathrm{C}$ & $\begin{array}{r}47.2 \% \\
(30.4-64.5 \%)\end{array}$ & $\mathrm{N} / \mathrm{C}$ \\
\hline
\end{tabular}

$\mathrm{N} / \mathrm{C}$, not calculable.

\section{Discussion}

In developing countries with low socio-economic differences and fragile healthcare system the COVID-19 monitoring and diagnosis are grave public health concerns. In accordance to WHO recommendation, rapid antigen testing should have $\geq 80 \%$ sensitivity and specificity of $\geq 97 \%$ [12]. Our study shows that Antigen based test (ECLIA) are somewhat in accordance with WHO to diagnose SARS-CoV-2 infection, in comparison to RT-PCR. They showed good performance characteristics, having sensitivity of $94.3 \%$, when test was processed in samples with high viral load. Noteworthy, this evaluation was executed in high prevalence setting of CoVID-19 hence the effectiveness of antigen-based test might be different in epidemiologically diverse conditions.

Antigen-based tests have emerged as one of the most convincing options, but there are few independent evaluations of their diagnostic results, therefore, their position in routine diagnostic workup is still unclear. Furthermore, in situation where viral load or replication is low during early and late infection this detection assay might miss some cases [12]. Consequently, to prevent community and hospital acquired infections it is very important to identify asymptomatic and pre-symptomatic individuals at an early stage having a potential to spread COVID-19 infection [13].

Despite their lower sensitivity than PCR, antigen-based tests improve TAT (turnaround time), which is critical for interrupting transmission chains and controlling the spread of the pandemic [14]. Moreover, the automated analyzer (Cobas e411) in our study can perform almost 80 antigen tests in 18 min which make it suitable for using in high throughput setting specially in this pandemic. Furthermore, some diagnostic algorithms endorse usage of such tests as the initial step for symptomatic patients in initial five days. Results of our study also support using Elecsys SARS-CoV-2 Antigen tests for this purpose. To best of our knowledge this was first study on performance of automated antigen assay (Elecsys SARS-CoV-2 Antigen). However a similar type of study was conducted on an automated antigen based immunoassay. In June 2020, Yosuke Hirotsu et al. evaluated the diagnostic performance of LUMIPULSE SARS-CoV-2 Ag, an automated antigen base testing kit, for CoVID-19 infection diagnosis to RT PCR [15]. Yosuke Hirotsu et al. reported that when the viral load in the samples was $>100$ copies LUMIPULSE SARS-CoV- $2 \mathrm{Ag}$ sensitivity was $100 \%$ comparing with RT-PCR that is slightly higher than our study sensitivity. The sensitivity gradually declined with decreasing viral load i.e. $60 \%$ sensitivity in samples with $10-100$ copies, $33 \%$ in samples with $1-10$ copies, and $26 \%$ in samples with less than 1 copy 
comparable to our study when sensitivity decreased gradually with decreasing viral load.

In this study, samples with $\mathrm{Ct}$ value $<25$, the sensitivity was $94.3 \%$ which was comparable to sensitivity of Sofia SARS Ag FIA [16]. Lorena Porte et al. reported sensitivity of Sofia SARS Ag FIA that was $93.8 \%$ in Ct value $\leq 25$ samples. Moreover, comparing results of our study with best commercially available rapid immunochromatographic kits, it was noted that automated SARS-CoV-2 antigen testing has comparable sensitivity $[6,17,18]$.

Porte et al. (2020) assessed SARS-CoV-2 antigen test based on ICT (fluorescence immunochromatographic) with 127 samples from nasopharynx and oropharynx including $82 \mathrm{pa}-$ tients already tested with RT-PCR, manifesting cumulative sensitivity of 93.9\% [19]. Antigen based diagnostic tests have illustrated their effectiveness to curtail the SARS-CoV-2 pandemic, amongst which Panbio rapid antigen test was evaluated in many instances [17]. However, in the perspective of the rising new waves of Corona virus pandemic, other manufacturers are providing extended variety of additional assays [20-22]. In samples with Ct value $\leq 25$, Panbio COVID-19 Ag Rapid Test Device perform best i.e. $>90 \%$ sensitivity and $>95 \%$ specificity $[5,17,18]$, which were similar to our findings. In addition, a comparable study in China, claimed 68\% sensitivity in 208 RT-PCR confirmed nasopharyngeal swab samples [23]. Furthermore, we demonstrated that both of these tests RT-PCR and antigen-based test can be carried on same VTM sample. One of the study's benefits is its consistency, as the same sample materials were utilized for the RT-PCR and automated antigen assay comparisons, eliminating the possibility of distribution errors from utilizing separate specimens.

Our study documents a limiting factor which is the decline in sensitivity of test i.e. 70.8 and $47.2 \%$ in Ct 26-30 and 31-35 respectively. Therefore, Antigen based diagnostic tests cannot be deemed as a substitute to RT-PCR but can be used in addition for diagnosing SARS-CoV-2 infection. WHO recommends cumulative frequency $\geq 80 \%$ and specificity of $\geq 97 \%$ [12]. Although Elecsys SARS-CoV-2 Ag assay does not fulfill completely WHO recommendation but at $\mathrm{Ct}<25$ the sensitivity reaches to $95 \%$. In samples of lower viral load this test might miss some cases. However, this shortcoming can be reduced with frequent testing of individuals, because antigen-based testing is cost effective and turnaround time is lesser than RT PCR as acknowledged by Mina et al. [24].

As a result, the information provided in this study is equally important for policymakers and strategic organizations at the national level, and it also responds to the global demand for reliable COVID-19 diagnostic testing. Finally, national testing methodologies must be validated on a regular basis, and the government must determine how to put these methods into practice using standard operating procedures.

\section{Conclusions}

In conclusion, based on the findings of our study the automated antigen testing (Elecsys SARS-CoV-2 Antigen) cannot replace molecular based testing like RT PCR. Elecsys SARS-CoV-2 Antigen should be used complementary to RT-PCR in testing algorithms. Also, during containment strategy this test can be used as a screening tool to identify super spreaders and infectious cases. In order to overcome its limitations in individuals with lower viral load, frequent testing strategy should be adopted while using automated antigen-based testing. Furthermore, additional clinical validation studies would be required to back up our suggestions. Our findings should not be extrapolated to other available rapid antigen detection tests without further research, and more prospective multicenter trials and meta-analyses are required to determine the utility of additional rapid antigen detection tests.

Acknowledgments: We acknowledge support provided by Roche Diagnostics Pakistan.

Research funding: None declared.

Author contributions: BI: collected the clinical and laboratory data and revised the final manuscript. MK: processed statistical analysis. NS: drafted the manuscript. MMD: Data analysis. VJ: Collected clinical and laboratory data. MS: Revised the final manuscript. All authors have accepted responsibility for the entire content of this manuscript and approved its submission.

Competing interests: Authors state no conflict of interest. Informed consent: Written informed consent was obtained from all individuals included in this study.

Ethical approval: The study was approved by the Research and Ethics Committee of Rehman Medical Institute, Peshawar.

Data availability: The datasets used and/or analyzed during the current study are available from the corresponding author on request.

\section{References}

1. WHO. Coronavirus disease (COVID-19) pandemic. World Health Organization; 2021. Available from: https://covid19.who.int/.

2. Saeed U, Uppal SR, Piracha ZZ, Rasheed A, Aftab Z, Zaheer H, et al. Evaluation of SARS-CoV-2 antigen-based rapid diagnostic kits in Pakistan: formulation of COVID-19 national testing strategy. Virol J 2021;18:1-5.

3. Merino P, Guinea J, Muñoz-Gallego I, González-Donapetry P, Galán JC, Antona N, et al. Multicenter evaluation of the Panbio ${ }^{\mathrm{TM}}$ COVID-19 rapid antigen-detection test for the diagnosis of SARS- 
CoV-2 infection. Clin Microbiol Infect 2021. https://doi.org/10. 1016/j.cmi.2021.02.001 [Epub ahead of print].

4. Perchetti GA, Huang M-L, Mills MG, Jerome KR, Greninger AL. Analytical sensitivity of the Abbott BinaxNOW COVID-19 Ag CARD. J Clin Microbiol 2021;59. https://doi.org/10.1128/JCM. 02880-20.

5. Fenollar F, Bouam A, Ballouche M, Fuster L, Prudent E, Colson P, et al. Evaluation of the Panbio Covid-19 rapid antigen detection test device for the screening of patients with Covid-19. J Clin Microbiol 2020. https://doi.org/10.1128/JCM.02589-20.

6. Salvagno GL, Gianfilippi G, Bragantini D, Henry BM, Lippi G. Clinical assessment of the Roche SARS-CoV-2 rapid antigen test. Diagnosis (Berl) 2021. https://doi.org/10.1515/dx2020-0154.

7. Laboratory biosafety guidance related to coronavirus disease 2019 (COVID-19): interim guidance, 12 February 2020. World Health Organization; 2020. [Online]. Available from: https:// apps.who.int/iris/handle/10665/331138.

8. Pallett SJ, Rayment M, Patel A, Fitzgerald-Smith SA, Denny SJ, Charani $E$, et al. Point-of-care serological assays for delayed SARS-CoV-2 case identification among health-care workers in the UK: a prospective multicentre cohort study. Lancet Respir Med 2020;8:885-94.

9. Health Department kpk. Daily Covid-19 SITREPS. Health Department Khyber Pukhtunkhwa; 2021. Available from: http:// healthkp.gov.pk/sitrep/view/283.

10. Roche Diagnostics. eLabDoc Elecsys SARS-CoV-2 antigen. Available from: https://pim-eservices.roche.com/eLD/web/pk/ en/products/CPS_000623?searchTerm=sars\&catalog=HealthcareProfessional\&orderBy=Relevance.

11. Lee S, Kim T, Lee E, Lee C, Kim H, Rhee H, et al. Clinical course and molecular viral shedding among asymptomatic and symptomatic patients with SARS-CoV-2 infection in a community treatment center in the Republic of Korea. JAMA Intern Med 2020;180:1447-52.

12. World Health Organization. Antigen-detection in the diagnosis of SARS-CoV-2 infection using rapid immunoassays: interim guidance, 11 September 2020. World Health Organization; 2020. Available from: https://apps.who.int/iris/handle/10665/ 334253.

13. Savvides C, Siegel R. Asymptomatic and presymptomatic transmission of SARS-CoV-2: a systematic review. medRxiv 2020;11. [Preprint]. https://doi.org/10.1101/2020.06.11. 20129072.
14. Broberg E, Ciancio B. Options for the use of rapid antigen tests for COVID-19 in the EU/EEA and the UK: European centre for disease prevention and control. Technical Report; 2020.

15. Hirotsu Y, Maejima M, Shibusawa M, Nagakubo Y, Hosaka K, Amemiya K, et al. Comparison of automated SARS-CoV-2 antigen test for COVID-19 infection with quantitative RT-PCR using 313 nasopharyngeal swabs, including from seven serially followed patients. Int J Infect Dis 2020;99:397-402.

16. Porte L, Legarraga P, Iruretagoyena M, Vollrath V, Pizarro G, Munita J, et al. Evaluation of two fluorescence immunoassays for the rapid detection of SARS-CoV-2 antigen-new tool to detect infective COVID-19 patients. Peerl 2021;9: e10801.

17. Albert E, Torres I, Bueno F, Huntley D, Molla E, Fernández-Fuentes MÁ, et al. Field evaluation of a rapid antigen test (Panbio ${ }^{\mathrm{TM}}$ COVID-19 Ag Rapid Test Device) for COVID-19 diagnosis in primary healthcare centres. Clin Microbiol Infect 2021;27:472.e7-e10.

18. Linares M, Pérez-Tanoira R, Carrero A, Romanyk J, Pérez-García F, Gómez-Herruz $P$, et al. Panbio antigen rapid test is reliable to diagnose SARS-CoV-2 infection in the first 7 days after the onset of symptoms. J Clin Virol 2020;133:104659.

19. Porte L, Legarraga P, Vollrath V, Aguilera X, Munita JM, Araos R, et al. Evaluation of a novel antigen-based rapid detection test for the diagnosis of SARS-CoV-2 in respiratory samples. Int J Infect Dis 2020;99:328-33.

20. Cerutti F, Burdino E, Milia MG, Allice T, Gregori G, Bruzzone B, et al. Urgent need of rapid tests for SARS CoV-2 antigen detection: evaluation of the SD-Biosensor antigen test for SARS-CoV-2. J Clin Virol 2020;132:104654.

21. Mak GC, Cheng PK, Lau SS, Wong KK, Lau C, Lam ET, et al. Evaluation of rapid antigen test for detection of SARS-CoV-2 virus. J Clin Virol 2020;129:104500.

22. Lambert-Niclot S, Cuffel A, Le Pape S, Vauloup-Fellous C, Morand-Joubert L, Roque-Afonso A-M, et al. Evaluation of a rapid diagnostic assay for detection of SARS CoV-2 antigen in nasopharyngeal swab. J Clin Microbiol 2020. https://doi.org/10. 1128/JCM.00977-20.

23. Diao B, Wen K, Chen J, Liu Y, Yuan Z, Han C, et al. Diagnosis of acute respiratory syndrome coronavirus 2 infection by detection of nucleocapsid protein. Clin Chim Acta 2020. https://doi.org/10. 1101/2020.03.07.20032524 [Epub ahead of print].

24. Mina MJ, Parker R, Larremore DB. Rethinking Covid-19 test sensitivity-a strategy for containment. N Engl J Med 2020;383: e120. 